\title{
Vertical quantum wire realized with double cleaved-edge overgrowth
}

Cite as: Appl. Phys. Lett. 89, 032102 (2006); https://doi.org/10.1063/1.2222347

Submitted: 18 April 2006 . Accepted: 13 June 2006. Published Online: 17 July 2006

S. F. Roth, H. J. Krenner, D. Schuh, M. Bichler, and M. Grayson

ARTICLES YOU MAY BE INTERESTED IN

Surface acoustic wave regulated single photon emission from a coupled quantum dotnanocavity system

Applied Physics Letters 109, 033105 (2016); https://doi.org/10.1063/1.4959079

Combined electrical transport and capacitance spectroscopy of a $\mathrm{MoS}_{2}$-LiNbO 3 field effect transistor

Applied Physics Letters 110, 023505 (2017); https://doi.org/10.1063/1.4973862

Ultrasonically assisted deposition of colloidal crystals

Applied Physics Letters 105, 031113 (2014); https://doi.org/10.1063/1.4891171

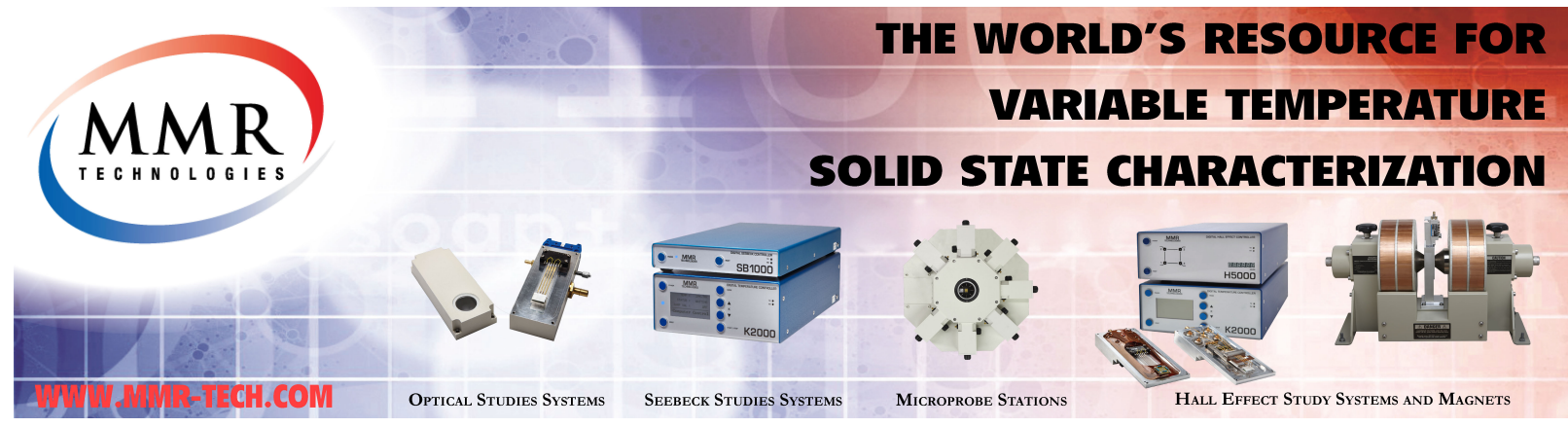




\title{
Vertical quantum wire realized with double cleaved-edge overgrowth
}

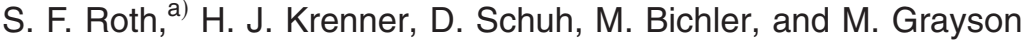 \\ Walter Schottky Institut, Technische Universität München, 85748 Garching, Germany
}

(Received 18 April 2006; accepted 13 June 2006; published online 17 July 2006)

\begin{abstract}
A quantum wire is fabricated on (001) GaAs at the intersection of two overgrown cleaves. The wire is contacted at each end to $n^{+}-\mathrm{GaAs}$ layers via two-dimensional (2D) leads. A side gate controls the density of the wire revealing conductance quantization. The step height is strongly reduced from $2 e^{2} / h$ due to the $2 \mathrm{D}$ lead series resistance. We characterize the $2 \mathrm{D}$ density and mobility for both cleave facets with four-point measurements. The density on the first facet is modulated by the substrate potential, depleting a $2 \mu \mathrm{m}$ wide strip that defines the wire length. Microphotoluminescence shows an extra peak consistent with one-dimensional electron states at the corner. (C) 2006 American Institute of Physics. [DOI: 10.1063/1.2222347]
\end{abstract}

An elegant technique for fabricating clean onedimensional (1D) quantum wires is cleaved-edge overgrowth (CEO), ${ }^{1}$ where the $1 \mathrm{D}$ wire arises at the intersection of a two-dimensional (2D) system and the perpendicular cleave plane. Ballistic transport, ${ }^{2,3}$ optical spectroscopy, 4,5 and lasing ${ }^{6,7}$ in such single-cleave wires have been investigated. Another advantage of CEO is that electron systems grown on the cleave facet can be modulated with atomic precision by the potential in the substrate. 2D CEO transport structures have been modulated with a superlattice potential, ${ }^{8}$ and with single ${ }^{9}$ and double tunnel barriers. ${ }^{10}$ The twin CEO capabilities of reducing the dimension and modulating the potential can be combined in double-cleave structures to create modulated 1D structures such as quantum dot molecules. ${ }^{11,12}$ To date, however, no transport structures have been implemented by double cleave due to the difficulty of fabricating electrical contacts.

This letter demonstrates a simple quantum wire in a double-cleave geometry with electrical contacts for transport measurements (Fig. 1). The wire develops at the intersection of two cleave planes in the accumulation edge of a depleted 2D electron system (2DES). Four-point measurements reveal the density and mobility of electrons in each overgrown facet, ${ }^{13}$ and when a $2 \mu \mathrm{m}$ wide low density strip in the first cleave plane is depleted, the conductance shows quantization, indicating 1D transport at the corner. The plateau steps are significantly reduced from $G_{0}=2 e^{2} / h$ due to the $2 \mathrm{D}$ lead series resistance. The quantum wire is also observed in photoluminescence as a second peak in the spectrum above the GaAs band gap energy.

Figure 1(a) shows the design of the vertical quantum wire. The substrate and first CEO are shown, with the second CEO taking place on the front facet. First, a (001) substrate is overgrown vertically with $1 \mu \mathrm{m} n^{+}$doped GaAs bottom contact, $2 \mu \mathrm{m}$ undoped GaAs, $2 \mu \mathrm{m} \mathrm{Al}_{0.3} \mathrm{Ga}_{0.7} \mathrm{As}$ (AlGaAs), another $2 \mu \mathrm{m}$ GaAs layer, and finally a $1 \mu \mathrm{m} n^{+}$top contact. This topmost layer is patterned with photolithography and partly etched away, leaving four $n^{+}$finger contacts ${ }^{13}$ which lead up to each cleave plane. Then the (110) facet is cleaved and overgrown, giving rise to the first 2DES (2DES I). The overgrowth consists of an $8 \mathrm{~nm}$ wide GaAs quantum well, a $50 \mathrm{~nm}$ AlGaAs spacer, Si $\delta$ doping, $500 \mathrm{~nm}$ AlGaAs, and a

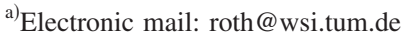

$n^{+}$side gate to control the $2 \mathrm{D}$ density. The $2 \mu \mathrm{m}$ wide AlGaAs substrate barrier modulates the density of 2DES I, both due to quantum confinement ${ }^{8}$ and because charged defects in AlGaAs can reduce the density next to the barrier. ${ }^{14,15}$ The reduced density region of $2 \mathrm{DES} \mathrm{I}$ is notated as $2 \mathrm{DES} \mathrm{I}^{*}$. To accumulate $1 \mathrm{D}$ states at the edge of $2 \mathrm{DES} \mathrm{I}$, the perpendicu$\operatorname{lar}(1 \overline{1} 0)$ cleave facet is overgrown with a modulation doped barrier: $20 \mathrm{~nm}$ AlGaAs spacer, Si $\delta$ doping, and $300 \mathrm{~nm}$ AlGaAs topped with a $20 \mathrm{~nm}$ GaAs cap. The accumulation

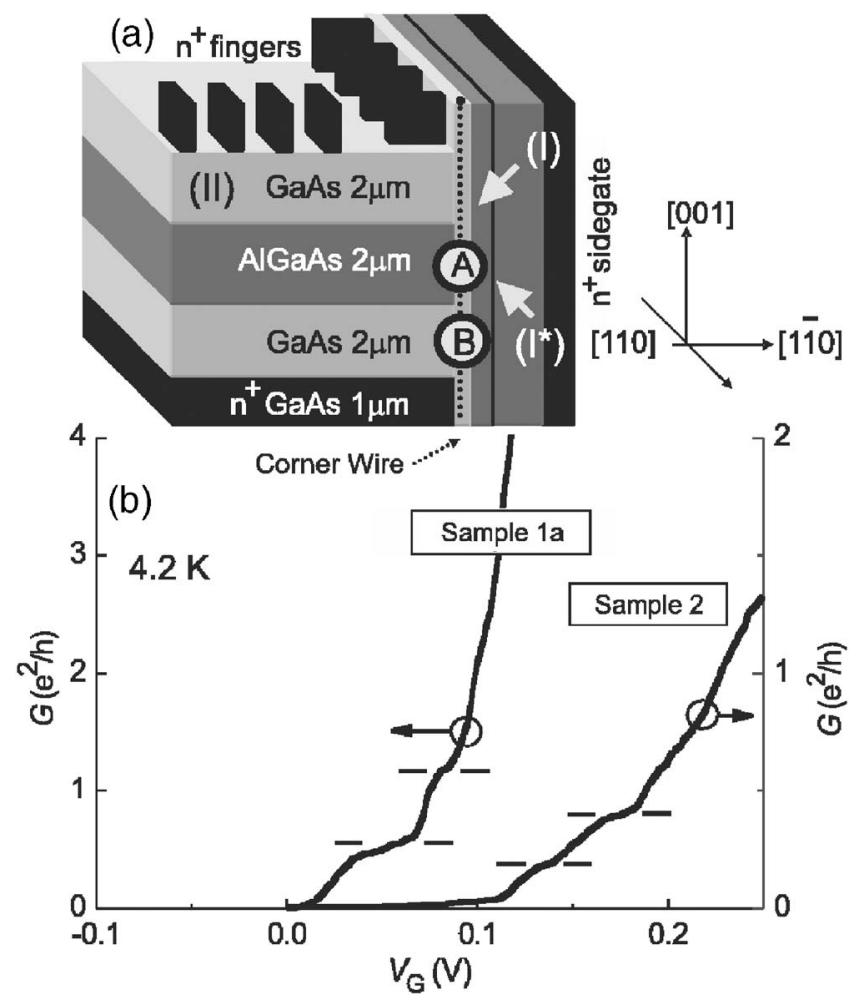

FIG. 1. (a) The double-cleave sample consists of a (001) grown substrate and a first (110) and second (1 $\overline{10})$ cleave, both overgrown with modulation doping. The $2 \mathrm{DES}$ regions from the two cleaves are indicated with (I) and (II), and the $2 \mu \mathrm{m}$ substrate barrier defines a reduced density strip in the first cleave denoted as $\left(I^{*}\right)$. The wire (dotted line) runs vertically at the corner of 2DES I and II and is contacted to the $n^{+}$top and bottom contacts. The second CEO is in the plane of the front facet. (b) The conductance of samples $1 \mathrm{a}$ and 2 exposes steps between $V_{G}=0$ and $0.2 \mathrm{~V}$, indicative of 1D conductance quantization. The $2 \mathrm{D}$ lead resistance suppresses the step height from $2 e^{2} / h$. 

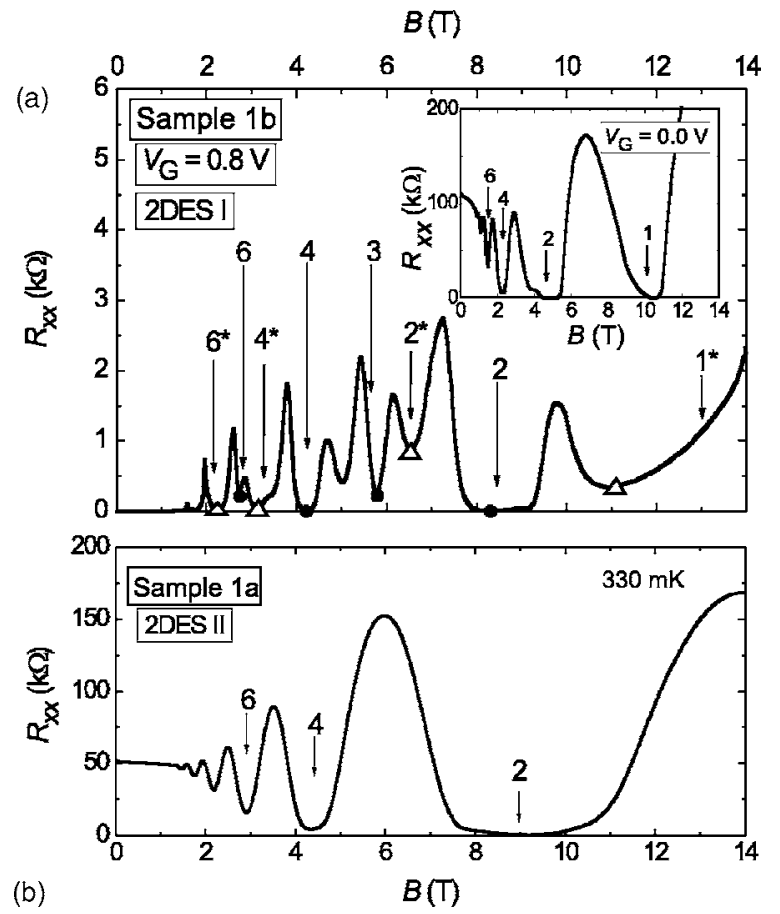

FIG. 2. Shubnikov-de Haas oscillations of (a) 2DES I for $V_{G}=0.8 \mathrm{~V}$ and $V_{G}=0.0 \mathrm{~V}$ (inset) and (b) 2DES II. A side gate tunes the density of 2DES I. Extra minima at $V_{G}=0.8 \mathrm{~V}$ are attributed to a second density in region 2DES I*. Filling factors are labeled $\nu$ for density $n_{\mathrm{I}}$ of 2DES I and $\nu^{*}$ for density $n_{\mathrm{I}^{*}}$ of 2DES I* Symbols indicate minima in the data and arrows indicate the theoretical position of minima as described in the text.

potential also induces a second 2DES (2DES II) at the second cleave plane, split by the substrate AlGaAs barrier.

Before measuring the wire, it is helpful to first characterize 2DES I, I*, and II with four-point magnetoresistance measurements using the $n^{+}$finger contacts. Standard lock-in techniques were used after illuminating the sample with a red light-emitting diode (LED). Figures 2(a) and 2(b) show the Shubnikov de Haas (SDH) oscillations measured at $330 \mathrm{mK}$ in a magnetic field perpendicular to the first and second cleave facets, respectively.

At a side gate bias $V_{G}=0.0 \mathrm{~V}$ [inset of Fig. 2(a)] only one density is apparent in the resistance oscillations on the first facet. The density and mobility $n_{\mathrm{I}}=2.15 \times 10^{11} \mathrm{~cm}^{-2}$ and $\mu_{\mathrm{I}}=2.6 \times 10^{4} \mathrm{~cm}^{2} / \mathrm{V} \mathrm{s}$ are presumed to correspond to $2 \mathrm{DES}$ I, since 2DES I* should be depleted. When $n_{\mathrm{I}}$ is increased with side gate bias to $V_{G}=0.8 \mathrm{~V}$ [Fig. 2(a)] the zero field resistance drops more than three orders of magnitude and additional minima appear. Both behaviors are evidence for the onset of conduction in 2DES I*. The low resistance arises because of a short to the bulk-doped bottom contact, and the additional minima arise from the lower density SdH oscillations in 2DES I*. The resistances $R_{\text {top-top }}$ between two finger contacts and $R_{\text {top-bottom }}$ from a finger to the bottom contact (Fig. 3 inset) confirm that two pinch-off conditions occur at $V_{G}=-0.1$ and $+0.3 \mathrm{~V}$, attributed to the depletions of 2DES I and 2DES I*, respectively. On the second cleave facet [Fig. $2(\mathrm{~b})]$, the density and mobility of $2 \mathrm{DES}$ II are $n_{\mathrm{II}}=4.3$ $\times 10^{11} \mathrm{~cm}^{-2}$ and $\mu_{\mathrm{II}}=2.9 \times 10^{4} \mathrm{~cm}^{2} / \mathrm{V} \mathrm{s}$, respectively.

We can quantify the densities of 2 DES I and I* at positive gate voltage by plotting Landau fans of the $R_{x x}$ minima versus $V_{G}$ (Fig. 3). The fit lines plot $B_{\nu}=n_{\mathrm{I}} h / e \nu$ (black) and $B_{\nu^{*}}=n_{\mathrm{I}}{ }^{*} h / e \nu^{*}$ (gray), where $B_{\nu}$ is the magnetic field of the $\nu$ th minima, and the densities $n_{\mathrm{I}}$ and $n_{\mathrm{I}^{*}}$ vary linearly with

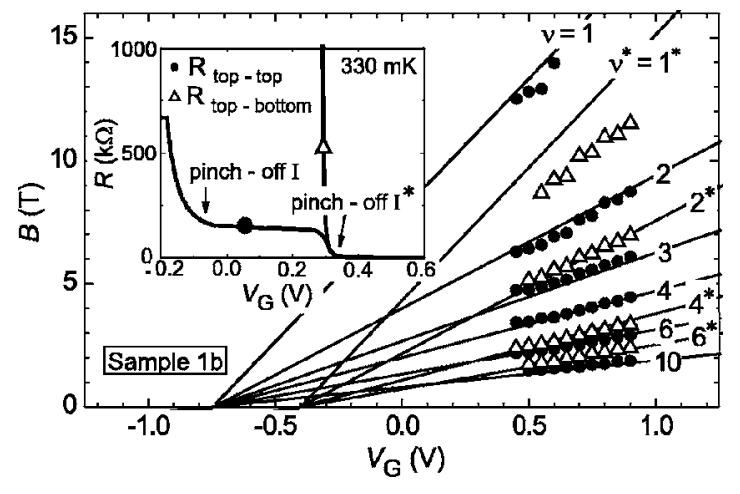

FIG. 3. (Inset) The gate voltage dependent resistances $R_{\text {top-top }}$ and $R_{\text {top-bottom }}$ demonstrate the pinch off of the low density central 2DES I* and of the high density 2DES I with decreasing $V_{G}$. The positions of the minima are plotted vs $V_{G}$ and fit to two Landau fans. A density difference of $\Delta n=n_{\mathrm{I}}-n_{\mathrm{I}^{*}}$ $=0.92 \times 10^{11} \mathrm{~cm}^{-2}$ is able to explain all the observed minima.

gate voltage with a fixed density difference $\Delta n=n_{\mathrm{I}}-n_{\mathrm{I}^{*}}{ }^{*}$ $=0.92 \times 10^{11} \mathrm{~cm}^{-2}$. The fits clearly identify all the observed minima. Just as in the inset of Fig. 3, the pinch-off of 2DES I occurs at $-0.4 \mathrm{~V}$ relative to the pinch off of $2 \mathrm{DES} \mathrm{I}{ }^{*}$, though differing cooldowns and illumination conditions shift the absolute gate voltage. At gate voltages where 2DES I* is depleted, transport in the 1D accumulation edge of 2DES I* can be investigated.

Conductance steps as a function of side gate bias in Fig. 1(b) are the principal evidence of $1 \mathrm{D}$ conduction in this structure. An ac voltage of $100 \mu \mathrm{V}$ is applied between a top finger contact and the bottom contact, and the current is measured with lock-in techniques at $17 \mathrm{~Hz}$. In Fig. 1(b) the conductance at $4.2 \mathrm{~K}$ of sample 1a after illumination is zero at a gate voltage of $V_{G}=0 \mathrm{~V}$, but rises to a plateau value of about $0.3 G_{0}$ between $V_{G}=0.03 \mathrm{~V}$ and $V_{G}=0.07 \mathrm{~V}$. A second plateau appears at $0.6 G_{0}$ between $V_{G}=0.07 \mathrm{~V}$ and $V_{G}=0.09 \mathrm{~V}$, indicating 1D transport. Sample 2 behaves similarly, with the conductance onset shifted to a slightly higher gate voltage and lower plateau values. The plateaus in both samples are strongly suppressed from $G_{0}$, due to a high $2 \mathrm{D}$ lead resistance as well as possible 2D-1D scattering at the accumulation edge., , $16,17^{2}$

Evidence of a quantum wire is also observed optically via microphotoluminescence ( $\mu$-PL). The sample is cooled down to $15 \mathrm{~K}$ in a flow cryostat, and a HeNe laser ( $\lambda$ $=632.8 \mathrm{~nm})$ is focused to a diameter of $1 \mu \mathrm{m}$ on the $(1 \overline{1} 0)$ facet [the front facet of Fig. 1(a)]. Figure 4 shows PL spectra recorded for low excitation densities $\left(P_{0}=200 \mathrm{~W} / \mathrm{cm}^{2}\right)$. As the spot is positioned exactly above 2DES I* [position A in Fig. 1(a)], a large peak appears in the PL signal at a detection energy $h \nu=1562 \mathrm{meV}$ and a smaller side peak at $1576 \mathrm{meV}$. When moving the laser spot along the [001] direction to rest above 2DES I, both peaks disappear [position B in Fig. 1(a)], because here no confinement for holes exists and only PL from the GaAs bulk is detected at the band gap energy of $1514 \mathrm{meV}$.

We calculate the interband absorption spectrum of an $8 \mathrm{~nm}$ GaAs quantum well embedded in an $\mathrm{AlGaAs}$ matrix. The lowest electron to heavy-hole transition was found to be at $E_{\mathrm{e}-\mathrm{hh}}=1574 \mathrm{meV}$, as indicated by the arrow in Fig. 4(a). Accordingly, we attribute the high energy peak to a transition between $2 \mathrm{D}$ electron and 2D hole states. We expect to find the 1D electron states shifted to lower energy, as shown by 

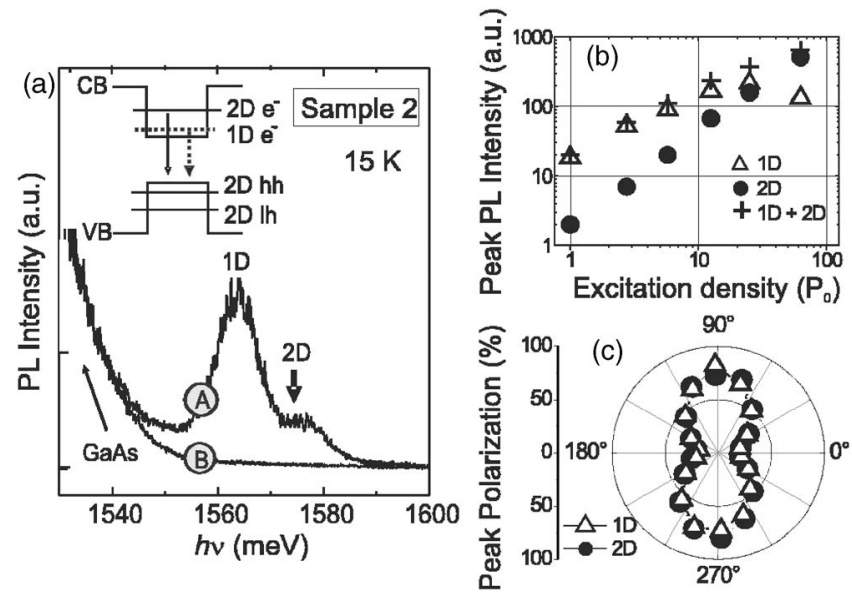

FIG. 4. (a) PL of a double-cleave sample measured on the square well (A) and the triangular well (B) (see Fig. 1). Two peaks appear at (A) and are attributed to a transition from $1 \mathrm{D}$ and $2 \mathrm{D}$ electron states to 2D heavy-hole states. The peaks disappear if the laser spot is moved to (B). The arrow indicates the calculated transition energy of the ground state. (b) At low laser powers only the $1 \mathrm{D}$ peak is visible. The $2 \mathrm{D}$ peak appears as the power is increased and electrons fill up higher states. When the lowest energy level is filled, the intensity of the 1D peak saturates. (c) Both peaks are strongly polarized as expected for transitions to heavy-hole states. The polarization angle $\theta=0^{\circ}$ is perpendicular to the quantum well growth direction.

the level diagram in Fig. 4(a). Consequently, we assign the low energy peak to a transition between bound 1D electron and a continuum of $2 \mathrm{D}$ heavy-hole states. ${ }^{5}$ No holes are confined to $1 \mathrm{D}$ in our structure. The energy difference of $14 \mathrm{meV}$ between the two peaks is the binding energy of the quantum wire and is in good agreement with Hartree calculations.

A final test for the existence of a quantum wire is excitation power dependent and polarization resolved spectroscopy. The results are presented in Figs. 4(b) and 4(c), respectively. For increasing excitation powers up to $100 P_{0}$ both peaks gain intensity and the $1 \mathrm{D}$ peak shows a clear saturation behavior in contrast to the 2D signal. This observation can be explained with a consecutive filling of the 1D and 2D levels. Due to the higher density of states, the 2D peak gains intensity superlinearly, the 1D peak sublinearly. For the sum of both signals an exponent of $0.97 \pm 0.05$ was found, demonstrating the single exciton nature and the decay to a common hole level. In Fig. 4(c) we plot the degree of polarization $I(\theta) /\left(I_{\perp}+I_{\|}\right)$of the $1 \mathrm{D}$ and $2 \mathrm{D}$ signals. For $\theta=0^{\circ}$ perpendicular to the quantum well growth direction of 2DES I, we find a maximum degree of polarization of $80 \%$ for both signals. Since the PL is detected perpendicular to the quantum well $(\mathrm{QW})$ growth direction heavy-hole and light-hole states can be distinguished. In contrast to light holes, heavy-hole transitions are polarized perpendicular to the QW growth direction due to the missing $z$ component of the central cell part of the wave function. The strong in-plane polarization of the 1D and 2D signals is a clear indication for a recombination of conduction band electrons with a heavy-hole state, ruling out a multiple peak structure from the light-hole energy spectrum. All four optical findings, the limited spatial area, the peak emission energy, the power dependent level filling, and the heavy-hole polarization selection rules, provide evidence for the formation of a bound 1D level for electrons below the $2 \mathrm{D}$ continuum.

In conclusion, we have fabricated a vertical quantum wire with twofold application of CEO. In conductance measurements, steps characteristic of 1D transport are observed. In optical investigations a peak in the PL spectrum on the low energy side of the quantum well signal is confirmed to arise from bound wire states with power and polarization dependent measurements. The vertical orientation of the wire makes it possible to fabricate samples with a $1 \mathrm{D}$ potential modulated by atomically precise barriers.

This work is supported by the Bundesministerium for Bildung und Forschung (BmBF) through Project No. 01BM469 and Deutsche Forschungsgemeinschaft Ab 35/4 and 35/5. The authors thank Jonathan J. Finley for assistance with PL measurements and Gerhard Abstreiter for discussions and continued support.

${ }^{1}$ L. Pfeiffer, K. West, H. Stormer, J. Eisenstein, K. Baldwin, and D. Gershoni, Appl. Phys. Lett. 56, 1697 (1997).

${ }^{2}$ A. Yacoby, H. L. Stormer, N. S. Wingreen, L. N. Pfeiffer, K. W. Baldwin, and K. W. West, Phys. Rev. Lett. 77, 4612 (1996).

${ }^{3}$ R. de Picciotto, H. L. Stormer, L. N. Pfeiffer, K. W. Baldwin, and K. W. West, Nature (London) 411, 51 (2001).

${ }^{4}$ J. Hasen, L. N. Pfeiffer, A. Pinczuk, S. He, K. West, and B. S. Dennis, Nature (London) 390, 54 (1997).

${ }^{5}$ A. R. Goni, L. N. Pfeiffer, K. W. West, A. Pinczuk, H. U. Baranger, and H. L. Stormer, Appl. Phys. Lett. 61, 1956 (1992).

${ }^{6}$ W. Wegscheider, L. N. Pfeiffer, M. M. Dignam, A. Pinczuk, K. W. West, S. L. McCall, and R. Hull, Phys. Rev. Lett. 71, 4071 (1993).

${ }^{7}$ Y. Hayamizu, M. Yoshita, S. Watanabe, H. Akiyama, L. N. Pfeiffer, and K. W. West, Appl. Phys. Lett. 81, 4937 (2002).

${ }^{8}$ R. A. Deutschmann, W. Wegscheider, M. Rother, M. Bichler, G. Abstreiter, C. Albrecht, and J. H. Smet, Phys. Rev. Lett. 86, 1857 (2001).

${ }^{9}$ W. Kang, H. Stormer, L. Pfeiffer, K. Baldwin, and K. West, Nature (London) 403, 59 (2000).

${ }^{10}$ F. Ertl, S. Roth, D. Schuh, M. Bichler, and G. Abstreiter, Physica E (Amsterdam) 22, 292 (2004).

${ }^{11}$ W. Wegscheider, G. Schedelbeck, G. Abstreiter, M. Rother, and M. Bichler, Phys. Rev. Lett. 79, 1917 (1997).

${ }^{12}$ G. Schedelbeck, W. Wegscheider, M. Bichler, and G. Abstreiter, Science 279, 1792 (1997).

${ }^{13}$ M. Grayson, S. F. Roth, Y. Xiang, F. Fischer, D. Schuh, and M. Bichler, Appl. Phys. Lett. 87, 212113 (2005).

${ }^{14}$ E. P. D. Poortere, Y. P. Shkolnikov, and M. Shayegan, Phys. Rev. B 67, 153303 (2003).

${ }^{15}$ S. F. Roth, M. Grayson, M. Bichler, D. Schuh, and G. Abstreiter, AIP Conf. Proc. 772, 915 (2005).

${ }^{16}$ R. de Picciotto, H. L. Stormer, A. Yacoby, L. N. Pfeiffer, K. W. Baldwin, and K. W. West, Phys. Rev. Lett. 85, 1730 (2000).

${ }^{17}$ Sample names: 03-04-05.1e (1a), 03-04-05.1c (1b), and 04-05-06.3c (2). 